medRxiv preprint doi: https://doi.org/10.1101/2021.12.21.21268205; this version posted December 30, 2021. The copyright holder for this

preprint (which was not certified by peer review) is the author/funder, who has granted medRxiv a license to display the preprint in

All rights reserved. No reuse allowed without permission.

Nunes et al. GAD-7 as a Marker of Inequity

\title{
General Anxiety Disorder-7 Questionnaire as a marker of low socioeconomic status and inequity
}

Julio C. Nunes, MDa , Megan K. Carroll, MS ${ }^{\mathrm{b}}$, Kenneth W. Mahaffey, MD ${ }^{\mathrm{a}}$, Robert M. Califf, MD ${ }^{\mathrm{b}}$, P. Murali Doraiswamy, MBBS ${ }^{\mathrm{c}, \mathrm{d}}$, Sarah Short, MPH ${ }^{\mathrm{b}}$, Svati H. Shah, MD, MHS e,f, Susan Swope, RN, MS a, Donna Williams, RN, MSN, MPH ${ }^{\text {a }}$, Adrian F. Hernandez, MD, MHS ${ }^{\mathrm{f} g}$, David S. Hong, $\mathrm{MD}^{\mathrm{h}}$

aStanford Center for Clinical Research, Stanford University School of Medicine, Stanford, CA, USA; bVerily Life Sciences (Alphabet), San Francisco, CA, USA; 'Department of Psychiatry and Behavioral Sciences, Duke University School of Medicine, Durham, NC, USA; ${ }^{d}$ Duke Institute for Brain Sciences, Duke University School of Medicine, Durham, NC, USA; eDuke Molecular Physiology Institute, Duke University School of Medicine, Durham, NC, USA; Division of Cardiology, Department of Medicine, Duke University School of Medicine, Durham, NC, USA; ' ${ }^{D}$ uke Clinical Research Institute, Duke University School of Medicine, Durham, NC, USA; hDepartment of Psychiatry and Behavioral Sciences, Stanford University School of Medicine, Stanford, CA, USA

\section{Corresponding author:}

Julio C. Nunes, MD

Stanford Center for Clinical Research

Stanford University

3180 Porter Dr.

Palo Alto, CA 94304

jcnunes@stanford.edu 
medRxiv preprint doi: https://doi.org/10.1101/2021.12.21.21268205; this version posted December 30, 2021. The copyright holder for this

preprint (which was not certified by peer review) is the author/funder, who has granted medRxiv a license to display the preprint in

All rights reserved. No reuse allowed without permission.

Nunes et al. GAD-7 as a Marker of Inequity

\section{ABSTRACT}

Background: The General Anxiety Disorder-7 (GAD-7) questionnaire is a standard tool used for screening and follow-up of patients with Generalized Anxiety Disorder (GAD). Although it is generally accepted that anxiety correlates with clinical and psychosocial stressors, precise quantitative data is limited on the relations among GAD-7, traditional biomarkers, and other measures of health. Even less is known about how GAD-7 relates to race, ethnicity, and socioeconomic status (SES).We determined how multiple demographic and socioeconomic data correlate with the participants' GAD-7 results when compared with laboratory, physical function, clinical, and other biological markers.

Methods: The Project Baseline Health Study (BHS) is a prospective cohort of adults representing several populations in the USA. We analyzed a deeply phenotyped group of 2502 participants from that study. Measures of interest included: clinical markers or history of medical diagnoses; physical function markers including gait, grip strength, balance time, daily steps, and echocardiographic parameters; psychometric measurements; activities of daily living; socioeconomic characteristics; and laboratory results.

Results: Higher GAD-7 scores were associated with female sex, younger age, and Hispanic ethnicity. Measures of low SES were also associated with higher scores, including unemployment, income $\leq \$ 25,000$, and $\leq 12$ years of education. After adjustment for 166 demographic, clinical, laboratory, and symptom characteristics, unemployment and overall higher SES risk scores were highly correlated with anxiety scores. Protective factors included Black race and older age.

Limitations: Correlations identified in this cross-sectional study cannot be used to infer causal relationships; further, we were not able to account for possible use of anxiety treatments by study participants.

Conclusions: These findings highlight the importance of understanding anxiety as a biopsychosocial entity. Clinicians and provider organizations need to consider both the physical manifestations of the disorder and their patients' social determinants of health when considering treatment pathways and designing interventions.

Key words: Generalized Anxiety Disorder-7; measures of health and disease; effective clinical intervention; social determinants of health 
medRxiv preprint doi: https://doi.org/10.1101/2021.12.21.21268205; this version posted December 30, 2021. The copyright holder for this preprint (which was not certified by peer review) is the author/funder, who has granted medRxiv a license to display the preprint in

All rights reserved. No reuse allowed without permission.

Nunes et al. GAD-7 as a Marker of Inequity

\section{INTRODUCTION}

Anxiety disorders are among the most common psychiatric diagnoses, with a lifetime prevalence of $32 \%$ in the United States. ${ }^{1}$ Worldwide, the World Health Organization estimates that about 300 million people are affected. ${ }^{2}$ In the United States, anxiety disorders may contribute $\$ 110$ million to $\$ 600$ million per million inhabitants in indirect costs to the health care system. ${ }^{3}$ They also carry a large individual cost-burden due to their high prevalence, and may lead to school absences, work underperformance, socialization impairment, and overall disability. ${ }^{4}$ Anxiety is closely related to multiple chronic disorders, such as cardiovascular disease, diabetes mellitus type 2, hypertension, arthritis, and chronic obstructive pulmonary disease..$^{5-7}$

Generalized Anxiety Disorder (GAD) is a chronic recurring subtype of anxiety, defined by persistent, excessive, difficult-to-control, and intrusive thoughts that may manifest comorbidly with somatic symptoms, sleep disturbances, and mental exhaustion. It frequently co-occurs with other psychiatric conditions, with at least $60 \%$ of the patients reporting having another disorder, mainly major depression, substance use disorder, attention deficithyperactivity disorder, and other types of anxiety. ${ }^{1}$

Spitzer et al. created the first standardized and widely available measurement tool for symptoms of GAD, the General Anxiety Disorder-7 (GAD7). ${ }^{8}$ Consisting of 7 questions scored from 0 to 3 , this tool is easily usable by clinicians of all specialties and levels. It has since been validated in primary care, general population, outpatient, and inpatient psychiatric settings. ${ }^{9-11}$ The tool's 7 components assess for 1) feeling nervous or on edge, 2) capacity to control worries and thoughts, 3) difficulty relaxing, 4) excessive worry about multiple themes, 5) restlessness, 6) irritability, and 7) constant worry or fear that unpleasant things will soon happen.

Several studies have examined possible correlations between higher degrees of anxiety and changes in laboratory measures, particularly biomarkers and peptides, related to serotonergic (e.g. 5-HT reuptake binding density, 5-HT plasma concentration), noradrenergic (e.g., platelet alpha-2 adrenergic peripheral binding density), and GABAergic (e.g., lymphocytes peripheral BDZ binding sites) systems. ${ }^{12-17}$ Oxidative and immunological systems are also of interest, and previous studies have shown increased levels of inflammatory markers and reactive oxygen radicals in patients experiencing intense degrees of anxiety. ${ }^{18-22}$ Vismara et al. recently reviewed and summarized the relationship between anxiety disorders and these biomarkers. ${ }^{23}$ However, despite these significant advances in understanding the physiology of anxiety, little has been published about how it correlates with wide-ranging population health markers, demographic characteristics, and social determinants of health.

The Baseline Health Study (BHS) ${ }^{24}$ is an ongoing prospective cohort study that aims to determine overall health biomarkers, biopsychosocial status, and demographics of a group of participants whose sex, race, and ethnicities are representative of the broader US adult population. In this study patients are deeply phenotyped, meaning that various demographic, laboratory, clinical, physical function, psychometric, and imaging findings are obtained annually during longitudinal follow-up visits. The BHS represents a unique opportunity to assess multiple types of anxiety correlates. 
medRxiv preprint doi: https://doi.org/10.1101/2021.12.21.21268205; this version posted December 30, 2021. The copyright holder for this preprint (which was not certified by peer review) is the author/funder, who has granted medRxiv a license to display the preprint in

All rights reserved. No reuse allowed without permission.

Nunes et al. GAD-7 as a Marker of Inequity

In this study, we focus on how multiple demographic and socioeconomic data correlate with GAD-7 results when compared with laboratory, physical function, clinical, and other biological markers. Our goal is to understand what role socioeconomic status (SES) and multiple other determinants of health may play in the biopsychosocial approach of anxiety disorders. Although some of the individual correlations have previously been reported, we add to the current knowledge by demonstrating the full range of these correlations in a single population, due to the comprehensive nature of data collection in the BHS.

\section{METHODS}

\section{The Baseline Health Study}

The Baseline Health Study is an ongoing multicenter longitudinal observational study. Multimodal data, including 166 different demographic, clinical, imaging, and laboratory measurements, were collected for 2502 adult participants. The BHS design has been previously described, including its data collection plan, inclusion and exclusion criteria, institutional review board regulation, and other main components. ${ }^{24}$

Study participants were recruited and enrolled via an online registration platform. The deeply phenotyped cohort used for this analysis was selected through an algorithm designed to produce a cohort that corresponds proportionately to U.S. adult population demographic data. Persons with various health statuses were included in the study, ranging from healthy controls to patients living with advanced disease. Due to the BHS methodology, the sampling was designed to over-represent persons with heart disease or cancer.

For this study, we performed a cross-sectional analysis that encompasses information of the baseline visit, when GAD-7 and the majority of the clinical data were collected. The GAD-7 was completed either at the in-person visit or digitally after the visit.

The BHS is funded by Verily Life Sciences (San Francisco, CA) and conducted by a collaborative group including Stanford University (Stanford, CA), Duke University (Durham, NC), and the California Health and Longevity Institute (Westlake Village, CA). The enrolling sites locations are Palo Alto, CA; Durham, NC; Kannapolis, NC; and Los Angeles, CA.

\section{Statis tical Analysis}

Demographic and clinical characteristics were described across the five GAD-7 severity categories $(0,1-4,5-9,10-14,15+)$, separately for women and men, in the population of participants who completed the GAD-7 survey at baseline. To test associations, the Cochrane-Armitage trend test was used for binary variables, while the Spearman rank correlation test was used for continuous variables. Given the exploratory nature of the study, we did not adjust for multiple tests. Data were considered non-missing if they were collected within 200 days of a participants' baseline in-person study visit.

\section{SES Risk Score}

Socioeconomic status variables were combined into a single "SES risk score" variable that was used in subsequent LASSO regressions and on Figure 2. The variable was a sum score of 5 individual items coded as 1/0: 1) reported high school education or less, 2) reported income $<\$ 25,000$, 3) marital status reported as unmarried, 4) employment status reported as not 
medRxiv preprint doi: https://doi.org/10.1101/2021.12.21.21268205; this version posted December 30, 2021. The copyright holder for this preprint (which was not certified by peer review) is the author/funder, who has granted medRxiv a license to display the preprint in

All rights reserved. No reuse allowed without permission.

Nunes et al. GAD-7 as a Marker of Inequity

working, and 5) insurance status reported as uninsured. A higher score was considered as having "higher risk" SES.

\section{Imputation of Missing Data}

All categorical predictors were converted into indicator variables via dummy coding. Multiple imputation by chained equations (MICE) was used to address missing data, as LASSO regression techniques require complete data on the input dataset. MICE assumes that all data are missing at random (MAR), meaning that the probability of a data point being missing depends only on observed data. The process generally consists of 4 steps: 1) a simple imputation (i.e., mean) is done for each missing value and used as placeholder, 2) placeholder imputations are set back to missing for a single "target" variable $X_{i}$,3) $X_{i}$ becomes the dependent variable and is regressed on all other variables in the imputation model, and 4) missing values in $X_{i}$ are replaced with predicted values from the model in step 3, and are then used in following regression models on variables with missing data. Steps 3-4 employ predictive mean matching in order to calculate the missing values of both continuous and categorical target variables; this ensures that missing data are assigned a value that has already been observed in the data. Steps 2-4 are repeated for each variable with missing data until all data are complete, a process that constitutes 1 "cycle." A preset number of cycles were completed, at which point a final imputed dataset was computed. This process was then repeated for a prespecified number of imputed datasets. ${ }^{25}$

\section{LASSO Regression}

LASSO procedures addressing multiply imputed data by using group methods (MI-LASSO) were conducted to select models of baseline characteristics that may be predictive of GAD-7 score (logarithm of GAD-7+1). All variables were standardized to allow for meaningful comparison of coefficients. In the MI-LASSO method, an $\mathrm{L}_{1}$-norm (the sum of the absolute values of the regression coefficients) penalization is placed on the regression model; this penalizes the inclusion of additional variables, and forces the coefficients of variables that do not contribute to the model to zero. In the presence of multiply imputed data, LASSO can produce different results for each imputed dataset; MI-LASSO fits models on all datasets jointly while considering each set of estimated coefficients $\left(\hat{\beta}_{1, \mathrm{j}}, \ldots, \hat{\beta}_{\mathrm{D}, \mathrm{j}}\right.$, where $\mathrm{D}$ is the number of imputed datasets) as a group, and applying the group LASSO penalty to the model. Shrinkage of the coefficients is controlled by the tuning parameter $(\lambda)$, which is optimized by selecting the model that minimizes the Bayesian Information Criterion (BIC). ${ }^{26}$ Analyses were performed using R v3.6.3 (R Foundation for Statistical Computing, Vienna, Austria). Data were imputed using the 'MICE' package v3.13.0. ${ }^{27}$ MI-LASSO regressions were run using the MI.LASSO function developed by Chen and Wang. ${ }^{26}$ Figures were created using the 'ggplot2' package v3.3.0. ${ }^{28}$

\section{RESULTS}

A total of 2,453 participants completed baseline GAD-7 assessments, of whom 1,343 (55\%) were female and 1,110 (45\%) were male. Associations between GAD-7 scores and demographic data are shown in Table 1. Younger age, Hispanic ethnicity, and identifying as other race (defined as one of: Native Hawaiian or Pacific Islander, American Indian or 
Table 1. Summary of demographic characteristics across GAD-7 severity scores at baseline, stratified by sex. Data are presented as N (\%) or median (IQR). P-values for trend were calculated with the use of Spearman Correlation or Cochrane-Armitage tests, where appropriate.

\begin{tabular}{|c|c|c|c|c|c|c|c|c|c|c|c|c|c|}
\hline \multirow[t]{2}{*}{ Demographics } & \multirow{2}{*}{$\begin{array}{l}\text { Overall } \\
\mathrm{N}=\mathbf{2 4 5 3}\end{array}$} & \multicolumn{6}{|c|}{ Female GAD-7 } & \multicolumn{6}{|c|}{ Male GAD-7 } \\
\hline & & $\begin{array}{c}0 \\
(\mathrm{~N}=\mathbf{4 0 4})\end{array}$ & $\begin{array}{c}1-4 \\
(N=531)\end{array}$ & $\begin{array}{c}5-9 \\
(\mathrm{~N}=257)\end{array}$ & $\begin{array}{c}10-14 \\
(N=103)\end{array}$ & $\begin{array}{c}15+ \\
(N=48)\end{array}$ & p-value & $\begin{array}{c}0 \\
(\mathrm{~N}=\mathbf{4 1 8})\end{array}$ & $\begin{array}{c}1-4 \\
(N=479)\end{array}$ & $\begin{array}{c}5-9 \\
(\mathrm{~N}=137)\end{array}$ & $\begin{array}{c}10-14 \\
(N=54)\end{array}$ & $\begin{array}{c}15+ \\
(\mathrm{N}=22)\end{array}$ & p-value \\
\hline Age, median (IQR) & $\begin{array}{c}50.0 \\
(35.3-64.1)\end{array}$ & $\begin{array}{c}57.2 \\
(43.3-68.7)\end{array}$ & $\begin{array}{c}49.7 \\
(35.3-62.4)\end{array}$ & $\begin{array}{c}47.5 \\
(34.3-58.2)\end{array}$ & $\begin{array}{c}44.1 \\
(32.7-56.0)\end{array}$ & $\begin{array}{c}42.8 \\
(30.0-54.2)\end{array}$ & $<0.0001$ & $\begin{array}{c}59.0 \\
(43.0-70.4)\end{array}$ & $\begin{array}{c}46.5 \\
(31.8-62.9)\end{array}$ & $\begin{array}{c}41.9 \\
(33.6-55.6)\end{array}$ & $\begin{array}{c}31.8 \\
(26.1-50.7)\end{array}$ & $\begin{array}{c}42.1 \\
(29.5-53.2)\end{array}$ & $<0.0001$ \\
\hline \multicolumn{14}{|l|}{ Race/Ethnicity } \\
\hline White, n (\%) & $1561(63.6)$ & $266(65.8)$ & $347(65.3)$ & $159(61.9)$ & $60(58.3)$ & $31(64.6)$ & 0.1842 & $272(65.1)$ & $300(62.6)$ & $90(65.7)$ & $28(51.9)$ & $8(36.4)$ & 0.0204 \\
\hline Black, n (\%) & $390(15.9)$ & $81(20.0)$ & $77(14.5)$ & $41(16.0)$ & $18(17.5)$ & $6(12.5)$ & 0.1680 & $62(14.8)$ & $68(14.2)$ & $18(13.1)$ & $10(18.5)$ & $9(40.9)$ & 0.0846 \\
\hline Asian, n (\%) & $254(10.4)$ & $29(7.2)$ & $49(9.2)$ & $25(9.7)$ & $6(5.8)$ & $3(6.2)$ & 0.9947 & $56(13.4)$ & $68(14.2)$ & $12(8.8)$ & $5(9.3)$ & $1(4.5)$ & 0.0943 \\
\hline $\begin{array}{l}\text { Native Hawaiian or } \\
\text { Pacific Islander, N (\%) }\end{array}$ & $27(1.1)$ & $3(0.7)$ & $7(1.3)$ & $5(1.9)$ & $0(0)$ & $0(0)$ & 0.6227 & $4(1.0)$ & $6(1.3)$ & $2(1.5)$ & $0(0)$ & $0(0)$ & 0.6063 \\
\hline $\begin{array}{l}\text { American Indian or } \\
\text { Alaska Native, n }(\%)\end{array}$ & $28(1.1)$ & $2(0.5)$ & $7(1.3)$ & $5(1.9)$ & $4(3.9)$ & $1(2.1)$ & 0.0142 & $4(1.0)$ & $3(0.6)$ & $1(0.7)$ & $1(1.9)$ & $0(0)$ & 0.6238 \\
\hline Other race, n (\%) & 193 (7.9) & $23(5.7)$ & $44(8.3)$ & $22(8.6)$ & $15(14.6)$ & 7 (14.6) & 0.0017 & $20(4.8)$ & $34(7.1)$ & $14(10.2)$ & $10(18.5)$ & $4(18.2)$ & $<0.0001$ \\
\hline Hispanic ethnicity, n (\%) & $279(11.3)$ & $38(9.4)$ & $58(10.9)$ & $36(14.0)$ & $23(22.3)$ & $12(25.0)$ & $<0.0001$ & $33(7.9)$ & $50(10.4)$ & $17(12.4)$ & $8(14.8)$ & $4(18.2)$ & 0.0146 \\
\hline \multicolumn{14}{|l|}{ Site } \\
\hline West Lake, n (\%) & $468(19.1)$ & 45 (11.1) & 97 (18.3) & $58(22.6)$ & $29(28.2)$ & $15(31.2)$ & $<0.0001$ & $60(14.4)$ & $114(23.8)$ & 30 (21.9) & $18(33.3)$ & $2(9.1)$ & 0.0069 \\
\hline Durham, n (\%) & 479 (19.5) & $101(25.0)$ & $112(21.1)$ & $50(19.5)$ & $16(15.5)$ & $7(14.6)$ & 0.0087 & 86 (20.6) & $66(13.8)$ & $19(13.9)$ & $14(25.9)$ & $8(36.4)$ & 0.7588 \\
\hline Kannapolis, n (\%) & $511(20.8)$ & 99 (24.5) & 111 (20.9) & 45 (17.5) & $22(21.4)$ & $16(33.3)$ & 0.6918 & $96(23.0)$ & $78(16.3)$ & $30(21.9)$ & $7(13.0)$ & $7(31.8)$ & 0.4171 \\
\hline Palo Alto, n (\%) & 995 (40.6) & 159 (39.4) & 211 (39.7) & $104(40.5)$ & $36(35.0)$ & $10(20.8)$ & 0.0912 & $176(42.1)$ & $221(46.1)$ & $58(42.3)$ & $15(27.8)$ & $5(22.7)$ & 0.0758 \\
\hline
\end{tabular}


medRxiv preprint doi: https://doi.org/10.1101/2021.12.21.21268205; this version posted December 30, 2021. The copyright holder for this preprint (which was not certified by peer review) is the author/funder, who has granted medRxiv a license to display the preprint in All rights reserved. No reuse allowed without permission.

Nunes et al. GAD-7 as a Marker of Inequity

Alaska Native, or Other race) were associated with higher GAD-7 scores for both men and women. The study's population racial-ethnic distribution represents that of the US census, with the exception of Hispanic persons, who are underrepresented (11.3\% of the study population compared with $18.5 \%$ according to 2019 estimates from the US Census).

Table 2 describes associations between markers of socioeconomic status and GAD-7 scores. Less education, lower income, unmarried status, not working for at least 1 year, and uninsured status were all associated with higher anxiety scores in both men and women. In general, higher GAD-7 scores were associated with lower SES across multiple types of measurement. 
Table 2. Summary of socioeconomic status across GAD-7 severity scores at base line, stratified by sex. Data are presented as N (\%) or median (IQR). P-values for trend were calculated with the use of Spearman Correlation or Cochrane-Armitage tests, where appropriate.

\begin{tabular}{|c|c|c|c|c|c|c|c|c|c|c|c|c|c|}
\hline \multirow[t]{2}{*}{ Socioeconomic Status } & \multirow{2}{*}{$\begin{array}{l}\text { Overall } \\
\mathrm{N}=\mathbf{2 4 5 3}\end{array}$} & \multicolumn{6}{|c|}{ Female GAD-7 } & \multicolumn{6}{|c|}{ Male GAD-7 } \\
\hline & & $\begin{array}{c}0 \\
(N=404)\end{array}$ & $\begin{array}{c}1-4 \\
(N=531)\end{array}$ & $\begin{array}{c}5-9 \\
(N=257)\end{array}$ & $\begin{array}{c}10-14 \\
(N=103)\end{array}$ & $\begin{array}{c}15+ \\
(N=48)\end{array}$ & p-value & $\begin{array}{c}0 \\
(N=418)\end{array}$ & $\begin{array}{c}1-4 \\
(N=479)\end{array}$ & $\begin{array}{c}5-9 \\
(\mathrm{~N}=137)\end{array}$ & $\begin{array}{c}10-14 \\
(\mathrm{~N}=54)\end{array}$ & $\begin{array}{c}15+ \\
(\mathbf{N}=22)\end{array}$ & p-value \\
\hline \multicolumn{14}{|l|}{ Education } \\
\hline High school or less, $\mathrm{n}(\%)$ & $176(7.2)$ & $28(6.9)$ & $32(6.0)$ & $17(6.6)$ & $15(14.6)$ & $9(18.8)$ & 0.0024 & $25(6.0)$ & $20(4.2)$ & $15(10.9)$ & $11(20.4)$ & $4(18.2)$ & $<0.0001$ \\
\hline Some college, n (\%) & $455(18.5)$ & $70(17.3)$ & $103(19.4)$ & $71(27.6)$ & $25(24.3)$ & $11(22.9)$ & 0.0076 & $65(15.6)$ & $70(14.6)$ & $24(17.5)$ & $10(18.5)$ & $6(27.3)$ & 0.2192 \\
\hline College, n (\%) & $634(25.8)$ & $120(29.7)$ & $178(33.5)$ & $68(26.5)$ & $24(23.3)$ & $11(22.9)$ & 0.0721 & $95(22.7)$ & $105(21.9)$ & $24(17.5)$ & $8(14.8)$ & $1(4.5)$ & 0.0192 \\
\hline $\begin{array}{l}\text { Graduate degree or higher, } \mathrm{n} \\
(\%)\end{array}$ & $670(27.3)$ & $123(30.4)$ & $140(26.4)$ & $58(22.6)$ & $13(12.6)$ & $4(8.3)$ & $<0.0001$ & $144(34.4)$ & $144(30.1)$ & $37(27.0)$ & $6(11.1)$ & $1(4.5)$ & $<0.0001$ \\
\hline \multicolumn{14}{|l|}{ Income } \\
\hline$<\$ 25,000, \mathrm{n}(\%)$ & $193(7.9)$ & $24(5.9)$ & $40(7.5)$ & $34(13.2)$ & $20(19.4)$ & $9(18.8)$ & $<0.0001$ & $20(4.8)$ & $24(5.0)$ & $6(4.4)$ & $10(18.5)$ & $6(27.3)$ & $<0.0001$ \\
\hline$\$ 25,000-50,000, \mathrm{n}(\%)$ & $256(10.4)$ & $43(10.6)$ & $52(9.8)$ & $39(15.2)$ & $15(14.6)$ & $9(18.8)$ & 0.0186 & $34(8.1)$ & $44(9.2)$ & $14(10.2)$ & $6(11.1)$ & $0(0.0)$ & 0.7462 \\
\hline$\$ 50,000-100,000, \mathrm{n}(\%)$ & $493(20.1)$ & $86(21.3)$ & $142(26.7)$ & $53(20.6)$ & $26(25.2)$ & $6(12.5)$ & 0.5255 & $70(16.7)$ & $77(16.1)$ & $24(17.5)$ & $7(13.0)$ & $2(9.1)$ & 0.4488 \\
\hline$\$ 100,000-150,000, \mathrm{n}(\%)$ & $308(12.6)$ & $57(14.1)$ & $61(11.5)$ & $28(10.9)$ & $2(1.9)$ & $2(4.2)$ & 0.0006 & $64(15.3)$ & $71(14.8)$ & 19 (13.9) & $2(3.7)$ & $2(9.1)$ & 0.0678 \\
\hline$\$ 150,000-200,000, \mathrm{n}(\%)$ & $197(8.0)$ & $35(8.7)$ & $50(9.4)$ & $16(6.2)$ & $5(4.9)$ & $2(4.2)$ & 0.0643 & $43(10.3)$ & $30(6.3)$ & $12(8.8)$ & $3(5.6)$ & $1(4.5)$ & 0.1106 \\
\hline$>\$ 200,000, \mathrm{n}(\%)$ & $358(14.6)$ & $64(15.8)$ & $77(14.5)$ & $27(10.5)$ & $8(7.8)$ & $3(6.2)$ & 0.0028 & 79 (18.9) & $75(15.7)$ & $20(14.6)$ & $5(9.3)$ & $0(0.0)$ & 0.0077 \\
\hline \multicolumn{14}{|l|}{ Marital Status } \\
\hline Married, n (\%) & $1054(43.0)$ & $179(44.3)$ & $242(45.6)$ & $97(37.7)$ & $31(30.1)$ & $11(22.9)$ & 0.0002 & $232(55.5)$ & $200(41.8)$ & $44(32.1)$ & $13(24.1)$ & $5(22.7)$ & $<0.0001$ \\
\hline Divorced, n (\%) & $180(7.3)$ & $42(10.4)$ & $49(9.2)$ & $30(11.7)$ & $12(11.7)$ & $4(8.3)$ & 0.7771 & $20(4.8)$ & $15(3.1)$ & $4(2.9)$ & $2(3.7)$ & $2(9.1)$ & 0.7563 \\
\hline $\begin{array}{l}\text { Formerly in long term } \\
\text { relationship, } \mathrm{n}(\%)\end{array}$ & $97(4.0)$ & $12(3.0)$ & $22(4.1)$ & $11(4.3)$ & $3(2.9)$ & $0(0.0)$ & 0.9021 & $16(3.8)$ & $24(5.0)$ & $5(3.6)$ & $3(5.6)$ & $1(4.5)$ & 0.6686 \\
\hline Living together, $\mathrm{n}(\%)$ & $205(8.4)$ & $26(6.4)$ & $46(8.7)$ & $31(12.1)$ & $9(8.7)$ & $8(16.7)$ & 0.0084 & $23(5.5)$ & $34(7.1)$ & $19(13.9)$ & $8(14.8)$ & $1(4.5)$ & 0.0049 \\
\hline $\begin{array}{l}\text { Never in long term } \\
\text { relationship, } \mathrm{n}(\%)\end{array}$ & $255(10.4)$ & $38(9.4)$ & $60(11.3)$ & $22(8.6)$ & $13(12.6)$ & $9(18.8)$ & 0.1884 & $26(6.2)$ & $54(11.3)$ & $21(15.3)$ & $9(16.7)$ & $3(13.6)$ & 0.0004 \\
\hline
\end{tabular}


Table 2. Summary of socioeconomic status across GAD-7 severity scores at base line, stratified by sex. Data are presented as N (\%) or median (IQR). P-values for trend were calculated with the use of Spearman Correlation or Cochrane-Armitage tests, where appropriate.

\begin{tabular}{|c|c|c|c|c|c|c|c|c|c|c|c|c|c|}
\hline \multirow[t]{2}{*}{ Socioeconomic Status } & \multirow{2}{*}{$\begin{array}{l}\text { Overall } \\
\mathrm{N}=\mathbf{2 4 5 3}\end{array}$} & \multicolumn{6}{|c|}{ Female GAD-7 } & \multicolumn{6}{|c|}{ Male GAD-7 } \\
\hline & & $\begin{array}{c}\mathbf{0} \\
(\mathrm{N}=\mathbf{4 0 4})\end{array}$ & $\begin{array}{c}1-4 \\
(N=531)\end{array}$ & $\begin{array}{c}\mathbf{5 - 9} \\
(\mathrm{N}=257)\end{array}$ & $\begin{array}{c}10-14 \\
(N=103)\end{array}$ & $\begin{array}{c}15+ \\
(N=48)\end{array}$ & p-value & $\begin{array}{c}0 \\
(N=418)\end{array}$ & $\begin{array}{c}1-4 \\
(N=479)\end{array}$ & $\begin{array}{c}5-9 \\
(\mathbf{N}=137)\end{array}$ & $\begin{array}{c}10-14 \\
(\mathrm{~N}=54)\end{array}$ & $\begin{array}{c}15+ \\
(\mathrm{N}=22)\end{array}$ & p-value \\
\hline Separated, n (\%) & $48(2.0)$ & $9(2.2)$ & $6(1.1)$ & $14(5.4)$ & $5(4.9)$ & $1(2.1)$ & 0.0378 & $6(1.4)$ & $4(0.8)$ & $3(2.2)$ & $0(0.0)$ & $0(0.0)$ & 0.8138 \\
\hline Widowed, n (\%) & $75(3.1)$ & $29(7.2)$ & $23(4.3)$ & $5(1.9)$ & $3(2.9)$ & $1(2.1)$ & 0.0025 & $3(0.7)$ & $9(1.9)$ & $2(1.5)$ & $0(0.0)$ & $0(0.0)$ & 0.4172 \\
\hline \multicolumn{14}{|l|}{ Employment Status } \\
\hline Employed for wages, $\mathrm{n}(\%)$ & $1147(46.8)$ & $189(46.8)$ & $273(51.4)$ & $121(47.1)$ & $40(38.8)$ & $20(41.7)$ & 0.1892 & $175(41.9)$ & $228(47.6)$ & $71(51.8)$ & $23(42.6)$ & $7(31.8)$ & 0.4526 \\
\hline Homemaker, n (\%) & $67(2.7)$ & $13(3.2)$ & $25(4.7)$ & $18(7.0)$ & $5(4.9)$ & $4(8.3)$ & 0.0372 & $0(0.0)$ & $0(0.0)$ & $1(0.7)$ & $1(1.9)$ & $0(0.0)$ & 0.0128 \\
\hline Unable to work, n (\%) & $65(2.6)$ & $1(0.2)$ & $11(2.1)$ & $11(4.3)$ & $13(12.6)$ & $4(8.3)$ & $<0.0001$ & $5(1.2)$ & $8(1.7)$ & $5(3.6)$ & $4(7.4)$ & $3(13.6)$ & $<0.0001$ \\
\hline Not working $\geq 1$ year, $\mathrm{n}(\%)$ & $49(2.0)$ & $5(1.2)$ & $11(2.1)$ & $8(3.1)$ & $4(3.9)$ & $2(4.2)$ & 0.0281 & $4(1.0)$ & $7(1.5)$ & $6(4.4)$ & $1(1.9)$ & $1(4.5)$ & 0.0279 \\
\hline Retired, n (\%) & $457(18.6)$ & $106(26.2)$ & $104(19.6)$ & $25(9.7)$ & $7(6.8)$ & $5(10.4)$ & $<0.0001$ & $121(28.9)$ & $72(15.0)$ & $12(8.8)$ & $5(9.3)$ & $0(0.0)$ & $<0.0001$ \\
\hline Student, $\mathrm{n}(\%)$ & $56(2.3)$ & $9(2.2)$ & $11(2.1)$ & $11(4.3)$ & $2(1.9)$ & $1(2.1)$ & 0.5223 & $5(1.2)$ & $13(2.7)$ & $2(1.5)$ & $2(3.7)$ & $0(0.0)$ & 0.2892 \\
\hline Not working $<1$ year, $\mathrm{n}(\%)$ & $58(2.4)$ & $11(2.7)$ & $9(1.7)$ & $7(2.7)$ & $5(4.9)$ & $1(2.1)$ & 0.4999 & $9(2.2)$ & $11(2.3)$ & $4(2.9)$ & $1(1.9)$ & $0(0.0)$ & 0.8428 \\
\hline Self-employed, n (\%) & $254(10.4)$ & $34(8.4)$ & $41(7.7)$ & $35(13.6)$ & $14(13.6)$ & $2(4.2)$ & 0.1543 & $46(11.0)$ & $61(12.7)$ & $14(10.2)$ & $4(7.4)$ & $3(13.6)$ & 0.7879 \\
\hline \multicolumn{14}{|l|}{ Health Insurance Status } \\
\hline Insured, n (\%) & $1809(73.7)$ & $321(79.5)$ & $436(82.1)$ & $192(74.7)$ & $67(65.0)$ & $32(66.7)$ & 0.0003 & $315(75.4)$ & $313(65.3)$ & $93(67.9)$ & $33(61.1)$ & $7(31.8)$ & $<0.0001$ \\
\hline Uninsured, n (\%) & $110(4.5)$ & $17(4.2)$ & $15(2.8)$ & $20(7.8)$ & $7(6.8)$ & $3(6.2)$ & 0.0391 & $11(2.6)$ & $24(5.0)$ & $7(5.1)$ & $2(3.7)$ & $4(18.2)$ & 0.0083 \\
\hline \multicolumn{14}{|l|}{ Smoking Status } \\
\hline Current smoker, n (\%) & $331(13.5)$ & $34(8.4)$ & $56(10.5)$ & $46(17.9)$ & $25(24.3)$ & $14(29.2)$ & $<0.0001$ & $43(10.3)$ & $62(12.9)$ & $23(16.8)$ & $17(31.5)$ & $11(50.0)$ & $<0.0001$ \\
\hline Former smoker, n (\%) & $539(22.0)$ & $73(18.1)$ & $119(22.4)$ & $55(21.4)$ & $21(20.4)$ & $8(16.7)$ & 0.6857 & $109(26.1)$ & $105(21.9)$ & $31(22.6)$ & $11(20.4)$ & $7(31.8)$ & 0.4639 \\
\hline Nonsmoker, $\mathrm{n}(\%)$ & $1583(64.5)$ & $297(73.5)$ & $356(67.0)$ & $156(60.7)$ & $57(55.3)$ & $26(54.2)$ & $<0.0001$ & $266(63.6)$ & $312(65.1)$ & $83(60.6)$ & $26(48.1)$ & $4(18.2)$ & 0.0005 \\
\hline
\end{tabular}


medRxiv preprint doi: https://doi.org/10.1101/2021.12.21.21268205; this version posted December 30, 2021. The copyright holder for this preprint (which was not certified by peer review) is the author/funder, who has granted medRxiv a license to display the preprint in

All rights reserved. No reuse allowed without permission.

Nunes et al. GAD-7 as a Marker of Inequity

Five datasets were imputed from the observed data using 5 cycles per imputation; the 5 datasets were simultaneously entered in the MI-LASSO model. Results from the full MILASSO regression models, which included 339 total predictors and was stratified by sex, are presented in Figure 1. Predictors classically described as somatic symptoms of anxiety per DSM-V criteria (e.g., headaches) were not included in the final model to allow for the observation of factors (e.g., symptoms of other medical conditions; sociodemographic data) not yet established as components of the syndrome of anxiety. A full list of variables entered into the MI-LASSO regression is available in Appendix A. In both men and women, memory change was the strongest positive predictor of GAD-7 severity. In women, the coefficient was more than twice that of the next strongest positive predictor (shortness of breath); in men, the coefficient was more than 3 times that of the next strongest positive predictor (muscle or joint pain). For women, we also observed positive associations between GAD-7 severity and other physical health-related symptoms (e.g., coughing up sputum, lightheadedness, diarrhea, and constipation), absolute monocyte levels, reporting Other race, and SES score. There were negative relationships between GAD-7 severity and both age and Black race. In men, there were positive associations between GAD-7 and other physical function measures including shortness of breath with exercise, joint pain or swelling, shortness of breath, and constipation. Similar to findings observed in women, GAD-7 severity decreased as age increased in men.

Figure 2 presents a heat map figure of associations between SES risk scores and GAD-7 severity, stratified by race. Overall associations between SES risk score and GAD-7 severity were significant for all races. Black participants tended to have higher concentrations in the high-SES risk, high-GAD-7 severity corner of the table; as SES risk score increased, the likelihood of having a higher GAD-7 severity score also increased. Black participants were also most likely to have an SES score of at least 3. White participants had higher concentrations in the low-SES risk, low GAD-7 corner of the table; although there was a similar trend toward higher GAD-7 scores as SES risk score increased, white participants had lower SES risk scores overall. Asian participants were more likely to have low SES risk, and few participants $(n=13)$ had a GAD-7 severity of at least 10 . There was no clear trend between SES risk score and GAD-7 severity for those who reported other races, but those participants tended to have lower SES risk scores overall.

\section{DISCUSSION}

Our study enrolled a large, deeply phenotyped cohort that allowed us to assess a broad array of anxiety correlates. Our findings confirm and highlight previous literature data regarding GAD-7 and different measurements of SES. We emphasize the importance of using GAD-7 with psychosocial contextual knowledge, as it raises concerns not only about psychological symptoms, but also about broader social determinants of health. Although some of the individual correlations are expected, their assembly highlights the importance of the biopsychosocial model when managing mental health disorders.

Previous studies have demonstrated how low income, unemployment, fewer years of education, lack of insurance, and smoking correlate with higher levels of anxiety. Hinz et al. validated the psychometric properties of the GAD-7 in Germany and found that those social determinants of health were correlated with higher anxiety scores. ${ }^{29}$ Finegan et al. (2020) 
medRxiv preprint doi: https://doi.org/10.1101/2021.12.21.21268205; this version posted December 30, 2021. The copyright holder for this preprint (which was not certified by peer review) is the author/funder, who has granted medRxiv a license to display the preprint in

All rights reserved. No reuse allowed without permission.

Nunes et al. GAD-7 as a Marker of Inequity

investigated the influence of socioeconomic deprivation and neighborhood violence on anxiety and found similar results but also observed that low neighborhood average income and high crime rates were likewise correlated with higher GAD-7 scores. ${ }^{30}$ Chen et al. reported similar findings in an analysis of 13,775 medical records. ${ }^{31}$

When designing interventions, clinicians and policy makers must consider socioeconomic issues, access to opportunities, and social context to better reduce anxiety. Different treatment modalities may be more effective if employed in a holistic approach that is sensitive to more than just biochemical matters or cognitive function. Furthermore, SES may limit the types of treatment and therapy patients may receive and thus, clinicians and health systems should be focused on innovative methods to enable people with anxiety to access the standard of care. ${ }^{32,33}$

Identifying as a person of Hispanic/Latinx ethnicity was correlated with higher GAD7 scores, a result consistent with previously published findings. Factors that may contribute to this correlation include higher rates of unemployment, job insecurity, lack of access to health care, distance from family, use of English as a second language, and systemic racism and prejudice. $^{34,35}$ Additionally, concerns about migration and visa status could lead certain ethnic groups to not enroll, which is reflected on the current underrepresentation of Latinx populations in our sample. We did not ask participants about their visa status and that is a limitation of this study. This aspect is important, as insecurity around residency status may also contribute to higher degrees of psychosocial distress among Hispanic populations . ${ }^{36,37}$

After adjustment for multiple factors, Black race was correlated with lower overall GAD7 scores, a finding consistent with previous studies suggesting that Black individuals may have higher levels of resiliency when facing adversity. ${ }^{38}$ However, when focusing on the relationships between race, SES risk score, and GAD-7 score (Figure 2), Black persons with high SES risk mostly have higher scores, suggesting that poor SES may be a significant associated factor in the development of anxiety for that group. This contrasts with white participants, who had higher levels of anxiety distributed across all levels of SES.

Nevertheless, this unexpected finding should be considered preliminary and warrants further investigation.

We also observed higher levels of anxiety among young unmarried women. These findings were previously reported in the literature and most recently updated during the COVID-19 pandemic. In studies of temporal trends and demographic disparities of mental health disorders, sex, age, and marital status were predictors of higher deterioration of mental health status, particularly when stricter social isolation measures were in place. ${ }^{39,40}$

The BHS is longitudinally assessing a large cohort of patients for markers of social, biological, clinical, and behavioral function; once it is completed, we will have an opportunity to better understand these relationships across time. Additionally, by the end of the study, race-and ethnicity-specific analyses may allow a more nuanced understanding of how social determinants of health shape the profile of anxiety in specific populations. The representation of each demographic group is a priority for the BHS study and its final population pool will reflect every race/ethnicity per the US census. The BHS is also an opportunity to understand the validity of behavioral data collection in the context of digital health. 
medRxiv preprint doi: https://doi.org/10.1101/2021.12.21.21268205; this version posted December 30, 2021. The copyright holder for this preprint (which was not certified by peer review) is the author/funder, who has granted medRxiv a license to display the preprint in All rights reserved. No reuse allowed without permission.

Nunes et al. GAD-7 as a Marker of Inequity

\section{Limitations}

A number of limitations to this study should be noted. Cross-sectional studies cannot determine causality. Therefore, this study is capable of demonstrating anxiety correlations but does not attempt to explain basic underlying causal effects. In addition, we lack details about anxiety treatments, which could be an unaccounted modifying factor. In future longitudinal follow-up studies, we plan to thoroughly consider behavioral treatments and medications and their associations.

\section{CONCLUSION}

In conclusion, higher GAD-7 scores were correlated with multiple measures across the spectrum of biological, clinical, behavioral and social measures. The relationship with SES is particularly striking, highlighting the importance of considering social determinants of health when designing interventions for anxiety. Focusing solely on the biological treatment of anxiety or the personal cognitive issues may have a limited effectiveness when compared with holistic approaches to care. Clinicians and policy makers are likely to be more effective if they are aware of social and contextual circumstances unique to the patient combined with social factors affecting groups. Additionally, when systems are set in place aiming to improve SES of individuals and populations, anxiety should be considered as a factor that may limit the capacity of these individuals to participate and respond to those interventions. 
medRxiv preprint doi: https://doi.org/10.1101/2021.12.21.21268205; this version posted December 30, 2021. The copyright holder for this preprint (which was not certified by peer review) is the author/funder, who has granted medRxiv a license to display the preprint in

All rights reserved. No reuse allowed without permission.

Nunes et al. GAD-7 as a Marker of Inequity

\section{Contributors}

JCN drafted and wrote the paper and performed the analysis. MKC contributed data or analysis tools, performed the analysis, wrote the paper. KWM conceived and designed the analysis, reviewed the paper. RMC conceived and designed the analysis, reviewed the paper. PMD conceived and designed the analysis, reviewed the paper. S Short contributed data or analysis tools, performed the analysis, reviewed the paper. SHS contributed data or analysis tools, reviewed the paper. S Swope collected the data, reviewed the paper. DW collected the data, reviewed the paper. AFH conceived and designed the analysis, reviewed the paper.

DSH conceived and designed the analysis, wrote and reviewed the paper, coordinated writing and analysis efforts.

\section{Declaration of Competing Inte rest}

None.

\section{Disclosures}

Dr. Califf is an employee of Verily Life Sciences and Google Health; he also serves as an advisory board/science advisory board member for Human Health and Potential (Singapore), Basking Biosciences, Launch and Scale Speedometer, and Medicxi Ventures. He is also a board member for AMYRIAD Pharma, Centessa Pharmaceuticals, Clinetic, One Fifteen, Portola, and Cytokinetics. Ms. Carroll and Ms. Short are employees of Verily Life Sciences. Dr. Hernandez notes external relationships with Amgen, AstraZeneca, Bayer, Biofourmis Singapore, Boehringer Ingelheim, Boston Scientific, Cytokinetics, Eidos Therapeutics, Eli Lilly, Intercept, Luitpold Pharmaceuticals, Merck, MyoKardia, Novartis, Pfizer, Relypsa, and Verily. Dr. Doraiswamy notes external relationships with Advera Health, Apollo Hospitals, Danone, Evidation Health, Live Love Laugh, Lumos Labs, MarvelBiome, Neuroglee, Transposon Therapeutics, Turtle Shell Technologies, UMethod, VitaKey, and Vivly. Dr. Shah reports external relationships with American Heart Association, AstraZeneca, Baylis Medical, Biosense Webster, Cardivia Medical, Lilly, McGraw-Hill Publishing, the National Institutes of Health, NewPace, and Project Baseline, LLC. Dr. Mahaffey's financial disclosures can be viewed at http://med.stanford.edu/profiles/kenneth-mahaffey.

\section{Acknowledgments}

Baseline Health Study Team: American Society of Clinical Oncology, Alexandria, VA, USA: Richard L. Schilsky. Duke University, School of Medicine, Durham, NC, USA: Jennifer Allen, MaryAnn Anderson, Kevin Anstrom, Lucus Araujo, Kristine Arges, Kaveh Ardalan, Bridget Baldwin, Suresh Balu, Mustafa R. Bashir, Manju Bhapkar, Robert Bigelow, Tanya Black, Rosalia Blanco, Gerald Bloomfield, Durga Borkar, Leah Bouk, Ebony Boulware, Nikki Brugnoni, Erin Campbell, Paul Campbell, Larry Carin, Tammy Jo Cassella, Tina Cates, Ranee Chatterjee Montgomery, Victoria Christian, John Choong, Michael CohenWolkowiez, Elizabeth Cook, Scott Cousins, Ashley Crawford, Nisha Datta, Melissa Daubert, James Davis, Jillian Dirkes, Isabelle Doan, Marie Dockery, P. Murali Doraiswamy, Pamela S. Douglas, Shelly Duckworth, Ashley Dunham, Gary Dunn, Ryan Ebersohl, Julie Eckstrand, Vivienne Fang, April Flora, Emily Ford, Lucia Foster, Elizabeth Fraulo, John French, Geoffrey S. Ginsburg, Cindy Green, Latoya Greene, Jeffrey Guptill, Donna Hamel, Jennifer 
medRxiv preprint doi: https://doi.org/10.1101/2021.12.21.21268205; this version posted December 30, 2021. The copyright holder for this preprint (which was not certified by peer review) is the author/funder, who has granted medRxiv a license to display the preprint in

All rights reserved. No reuse allowed without permission.

Nunes et al. GAD-7 as a Marker of Inequity

Hamill, Chris Harrington, Rob Harrison, Lauren Hedges, Brooke Heidenfelder, Adrian F. Hernandez, Cindy Heydary, Tim Hicks, Lina Hight, Deborah Hopkins, Erich S. Huang, Grace Huh, Jillian Hurst, Kelly Inman, Gemini Janas, Glenn Jaffee, Janace Johnson, Tiffanie Keaton, Michel Khouri, Daniel King, Jennifer Korzekwinski, Lynne H. Koweek, Anthony Kuo, Lydia Kwee, Dawn Landis, Rachele Lipsky, Desiree Lopez, Carolyn Lowry, Kelly Marcom, Keith Marsolo, Paige McAdams, Shannon McCall, , Robert McGarrah, John McGugan, Dani Mee, Sabrena Mervin-Blake, Prithu Mettu, Mathias Meyer, Justin Meyers, Calire N. Miller, Rebecca Moen, Lawrence H. Muhlbaier, Michael Murphy, Ben Neely, L. Kristin Newby, Jayne Nicoldson, Hoang Nguyen, Maggie Nguyen, Lori O’Brien, Sumru Onal, Jeremey O'Quinn, David Page, Neha J. Pagidipati, Kishan Parikh, Sarah R. Palmer, Bray Patrick-Lake, Brenda Pattison, Michael Pencina, Eric D. Peterson, Jon Piccini, Terry Poole, Tom Povsic, Alicia Provencher, Dawn Rabineau, Annette Rich, Susan Rimmer, Fides Schwartz, Angela Serafin, Nishant Shah, Svati Shah, Kelly Shields, Steven Shipes, Peter Shrader, Jon Stiber, Lynn Sutton, Geeta Swamy, Betsy Thomas, Sandra Torres, Debara Tucci, Anthony Twisdale, Susan A. Whitney, Robin Williamson, Lauren Wilverding, Charlene A. Wong, Lisa Wruck. Ellen Young Gemini Group, USA: Jane Perlmutter. Health Collaboratory and Cancer 101, New York, NY, USA: Sarah Krug. Rare Dots, Inc., USA: S. Whitney Bowman-Zatzkin. Society of Participatory Medicine, USA: Sarah Krug. Stanford University, School of Medicine, Stanford, CA, USA: Themistocles Assimes, Vikram Bajaj, Maxwell Cheong, Millie Das, Manisha Desai, Alice C. Fan, Dominik Fleischmann, Sanjiv S. Gambhir, Garry Gold, Francois Haddad, David Hong, Curtis Langlotz, Yaping J. Liao, Rong Lu, Kenneth W. Mahaffey, David Maron, Rebecca McCue, Rajan Munshi, Fatima Rodriguez, Sumana Shashidhar, George Sledge, Susie Spielman, Ryan Spitler, Sue Swope, Donna Williams, Julio C. Nunes. University of Florida, College of Medicine, Gainesville, FL, USA: Carl J Pepine. University of Missouri, Children's Mercy Hospital, Kansas City, MO, USA: John D Lantos. University of Texas, Dell Medical School, Austin, TX, USA: Michael Pignone. University of Washington, Department of Biostatistics, Seattle, WA, USA: Patrick Heagerty. Vanderbilt University, School of Medicine, Nashville, TN, USA: Laura Beskow, Gordon Bernard. Verily Inc., South San Francisco, CA, USA: Kelley Abad, Giulia Angi, Robert M. Califf, Lawrence Deang, Joy Huynh, Manway Liu, Cherry Mao, Michael Magdaleno, William J. Marks, Jr., Jessica Mega, David Miller, Nicole Ong, Darshita Patel, Vanessa Ridaura, Scarlet Shore, Sarah Short, Michelle Tran, Veronica Vu, Celeste Wong. Harvard University, School of Medicine, Boston, MA, USA: Robert C. Green. Google Inc., Mountain View, CA, USA: John Hernandez. California Health and Longevity Institute, Westlake Village, CA, USA: Jolene Benge, Gislia Negrete, Gelsey Sierra, Terry Schaack

\section{Data availability}

The Baseline Study data will be available to qualified researchers for exploratory analysis after the data are adequately curated and initial planned primary manuscripts are written. Qualified external researchers will be able to apply through applications reviewed by the Proposal Review and Publications Committee and Scientific Executive Committee. 
medRxiv preprint doi: https://doi.org/10.1101/2021.12.21.21268205; this version posted December 30, 2021. The copyright holder for this preprint (which was not certified by peer review) is the author/funder, who has granted medRxiv a license to display the preprint in All rights reserved. No reuse allowed without permission.

Nunes et al. GAD-7 as a Marker of Inequity

\section{Funding}

The Baseline Health Study and this analysis were funded by Verily Life Sciences, South San Francisco, CA. This analysis was funded by Verily Life Sciences (South San Francisco, CA) in partnership with Stanford University and Duke University.

\section{Role of the funding source}

The employees from funding source contributed to the data analyses, interpretation, editing of the manuscript and are coauthors. The final decision to submit the manuscript was made by the academic authors. 
medRxiv preprint doi: https://doi.org/10.1101/2021.12.21.21268205; this version posted December 30, 2021. The copyright holder for this

preprint (which was not certified by peer review) is the author/funder, who has granted medRxiv a license to display the preprint in

All rights reserved. No reuse allowed without permission.

Nunes et al. GAD-7 as a Marker of Inequity

\section{REFERENCES}

1. Kessler RC, Berglund P, Demler O, et al. Lifetime prevalence and age-of-onset distributions of DSM-IV disorders in the National Comorbidity Survey Replication. Arch Gen Psychiatry. 2005; 62:593-602.

2. Kessler RC, Angermeyer M, Anthony JC, R DG, et al. Lifetime prevalence and ageof-onset distributions of mental disorders in the World Health Organization's World Mental Health Survey Initiative. World Psychiatry. 2007; 6:168-176.

3. Konnopka A, Leichsenring F, Leibing E, et al. Cost-of-illness studies and costeffectiveness analyses in anxiety disorders: a systematic review. J Affect Disord 2009; 114:14-31.

4. Swinson R, Antony MM, Bleau P, et al. Management of anxiety disorders. Can J Psychiatry. 2006; 51 Suppl 2:1-92S.

5. Sareen J, Cox BJ, Clara I, et al. The relationship between anxiety disorders and physical disorders in the US National Comorbidity Survey. Depress Anxiety 2005; 21:193-202.

6. $\quad$ Smyth R: Depression in physical illness. J R Coll Physicians Edinb 2009; 39:337342.

7. Olver JS, Hopwood MJ: Depression and physical illness. Med J Aust 2013; 199(Suppl):S9-S12.

8. Spitzer RL, Kroenke K, Williams JB, et al. A brief measure for assessing generalized anxiety disorder: the GAD-7. Arch Intern Med. 2006; 166(10):1092-7.

9. Ruiz MA, Zamorano E, Garcia-Campayo J, et al. Validity of the GAD-7 scale as an outcome measure of disability in patients with generalized anxiety disorders in primary care. Jou Affec Disord. 2011; 128:277-286.

10. Löwe B, Decker O, Müller S, et al. Validation and standardization of the Generalized Anxiety Disorder Screener (GAD-7) in the general population. Med Care. 2008; 46(3):266-74.

11. Johnson SU, Ulvenes PG, Øktedalen T, et al. Psychometric Properties of the General Anxiety Disorder 7-Item (GAD-7) Scale in a Heterogeneous Psychiatric Sample. Front Psychol. 2019; 10:1713.

12. Iny LJ, Pecknold J, Suranyi-Cadotte BE, et al. Studies of a neurochemical link between depression, anxiety, and stress from $[3 \mathrm{H}]$ imipramine and $[3 \mathrm{H}]$ paroxetine binding on human platelets. Biol. Psychiatry. 1994; 36:251-291

13. Schneider LS, Munjack D, Severson JA, et al. Platelet [3H]Imipramine binding in generalized anxiety disorder, panic disorder, and agoraphobia with panic attacks. Biol. Psychiatry. 1987; 22:59-66. 
medRxiv preprint doi: https://doi.org/10.1101/2021.12.21.21268205; this version posted December 30, 2021. The copyright holder for this preprint (which was not certified by peer review) is the author/funder, who has granted medRxiv a license to display the preprint in

All rights reserved. No reuse allowed without permission.

Nunes et al. GAD-7 as a Marker of Inequity

14. Cameron OG, Smith CB, Lee MA, et al. Adrenergic status in anxiety disorders: Platelet alpha2-adrenergic receptor binding, blood pressure, pulse, and plasma catecholamines in panic and generalized anxiety disorders patients and in normal subjects. Biol. Psychiatry. 1990; 28:3-20.

15. Hernández E, Lastra S, Urbina M, et al. Serotonin, 5-hydroxyindoleacetic acid and serotonin transporter in blood peripheral lymphocytes of patients with generalized anxiety disorder. Int. Immunopharmacol. 2002; 2:893-900.

16. Weizman R, Tanne Z, Granek M, et al. Peripheral benzodiazepine binding sites on platelet membranes are increased during diazepam treatment of anxious patients. Eur. J. Pharmacol. 1987; 138:289-292.

17. Sevy S, Papadimitriou GN, Surmont DW, et al. Noradrenergic function in generalized anxiety disorder, major depressive disorder, and healthy subjects. Biol. Psychiatry. 1989; 25:141-152.

18. Costello H, Gould RL, Abrol E, et al. Systematic review and meta-analysis of the association between peripheral inflammatory cytokines and generalised anxiety disorder. BMJ Open. 2019; 9:e027925.

19. Maes M, Bonifacio KL, Morelli NR, et al. Generalized Anxiety Disorder (GAD) and Comorbid Major Depression with GAD Are Characterized by Enhanced Nitrooxidative Stress, Increased Lipid Peroxidation, and Lowered Lipid-Associated Antioxidant Defenses. Neurotox. Res. 2018; 34:489-510.

20. Findıklı E, Camkurt MA, Izci F, et al. The Diagnostic Value of Malondialdehyde, Superoxide Dismutase and Catalase Activity in Drug Naïve, First Episode, NonSmoker Generalized Anxiety Disorder Patients. Clin. Psychopharmacol. Neurosci. 2018; 16:88-94.

21. Emhan A, Selek S, Bayazit H, et al. Evaluation of oxidative and antioxidative parameters in generalized anxiety disorder. Psychiatry Res. 2015; 230:806-810.

22. Ercan AC, Bahceci B, Polat S, et al. Oxidative status and prolidase activities in generalized anxiety disorder. Asian J. Psychiatry. 2017; 25:118-122.

23. Vismara M, Girone N, Cirnigliaro G, et al. Peripheral Biomarkers in DSM-5 Anxiety Disorders: An Updated Overview. Brain Sci. 2020; 10(8):564.

24. Arges K, Assimes T, Bajaj V, et al. The Project Baseline Health Study: a step towards a broader mission to map human health. NPJ Digit Med. 2020; 3:84.

25. Azur MJ, Stuart EA, Frangakis C, et al. Multiple imputation by chained equations: what is it and how does it work?. Int J Methods Psychiatr Res. 2011; 20(1):40-49.

26. Chen Q, Wang S. Variable selection for multiply-imputed data with application to dioxin exposure study. Stat Med. 2013; 32(21):3646-59. 
medRxiv preprint doi: https://doi.org/10.1101/2021.12.21.21268205; this version posted December 30, 2021. The copyright holder for this

preprint (which was not certified by peer review) is the author/funder, who has granted medRxiv a license to display the preprint in

All rights reserved. No reuse allowed without permission.

Nunes et al. GAD-7 as a Marker of Inequity

27. Stef van Buuren, Karin Groothuis-Oudshoorn. MICE: Multivariate Imputation by Chained Equations in R. Journal of Statistical Software. 2011; 45(3):1-67. Available at: https://www.jstatsoft.org/v45/i03/.

28. H. Wickham. ggplot2: Elegant Graphics for Data Analysis. Springer-Verlag, New York, 2016.

29. Hinz A, Klein AM, Brähler E, et al. Psychometric evaluation of the Generalized Anxiety Disorder Screener GAD-7, based on a large German general population sample. J Affect Disord. 2017 Mar 1;210:338-344.

30. Finegan M, Firth N, and Delgadillo J. Adverse impact of neighbourhood socioeconomic deprivation on psychological treatment outcomes: the role of arealevel income and crime. Psychother Res. 2020 Apr;30(4):546-554.

31. Chen R, Kessler RC, Sadikova E, et al. Racial and ethnic differences in individuallevel and area-based socioeconomic status and 12-month DSM-IV mental disorders. J Psychiatr Res. 2019 Dec;119:48-59.

32. Giebel C, Corcoran R, Goodall M, et al. Do people living in disadvantaged circumstances receive different mental health treatments than those from less disadvantaged backgrounds? BMC Public Health. 2020 May 11;20(1):651.

33. Evans-Lacko S, Aguilar-Gaxiola S, Al-Hamzawi A, et al. Socio-economic variations in the mental health treatment gap for people with anxiety, mood, and substance use disorders: results from the WHO World Mental Health (WMH) surveys. Psychol Med. 2018 Jul;48(9):1560-1571.

34. Alvarez K, Fillbrunn M, Green JG, Jackson JS, Kessler RC, McLaughlin KA, Sadikova E, Sampson NA, Alegría M. Race/ethnicity, nativity, and lifetime risk of mental disorders in US adults. Soc Psychiatry Psychiatr Epidemiol. 2019 May;54(5):553-565.

35. Georgiades K, Paksarian D, Rudolph KE, Merikangas KR. Prevalence of Mental Disorder and Service Use by Immigrant Generation and Race/Ethnicity Among U.S. Adolescents. J Am Acad Child Adolesc Psychiatry. 2018 Apr;57(4):280-287.e2.

36. Alif A, Nelson BS, Stefancic A, Ahmed R, Okazaki S. Documentation status and psychological distress among New York City community college students. Cultur Divers Ethnic Minor Psychol. 2020 Jan;26(1):11-21

37. Ross J, Hua S, Perreira KM, Hanna DB, Castañeda SF, Gallo LC, Penedo FJ, Tarraf W, Hernandez R, Vega Potler N, Talavera GA, Daviglus ML, Gonzalez F 2nd, Kaplan RC, Smoller-Wassertheil S. Association between immigration status and anxiety, depression, and use of anxiolytic and antidepressant medications in the Hispanic Community Health Study/Study of Latinos. Ann Epidemiol. 2019 Sep;37:17-23 
medRxiv preprint doi: https://doi.org/10.1101/2021.12.21.21268205; this version posted December 30, 2021. The copyright holder for this preprint (which was not certified by peer review) is the author/funder, who has granted medRxiv a license to display the preprint in All rights reserved. No reuse allowed without permission.

Nunes et al. GAD-7 as a Marker of Inequity

38. Assari S. Number of Chronic Medical Conditions Fully Mediates the Effects of Race on Mortality; 25-Year Follow-Up of a Nationally Representative Sample of Americans. J. Racial and Ethnic Health Disparities 4, 623-631 (2017). https://doi.org/10.1007/s40615-016-0266-4

39. Santabárbara J, Lasheras I, Lipnicki DM, Bueno-Notivol J, Pérez-Moreno M, LópezAntón R, De la Cámara C, Lobo A, Gracia-García P. Prevalence of anxiety in the COVID-19 pandemic: An updated meta-analysis of community-based studies. Prog Neuropsychopharmacol Biol Psychiatry. 2021 Jul 13;109:110207. doi:

10.1016/j.pnpbp.2020.110207. Epub 2020 Dec 15. PMID: 33338558; PMCID: PMC7834650.

40. Kantor BN, Kantor J. Mental Health Outcomes and Associations During the COVID19 Pandemic: A Cross-Sectional Population-Based Study in the United States. Front Psychiatry. 2020 Dec 9;11:569083. 
medRxiv preprint doi: https://doi.org/10.1101/2021.12.21.21268205; this version posted December 30, 2021. The copyright holder for this preprint (which was not certified by peer review) is the author/funder, who has granted medRxiv a license to display the preprint in All rights reserved. No reuse allowed without permission.

Nunes et al. GAD-7 as a Marker of Inequity

\section{Figure Legends}

Figure 1. Factors associated with GAD-7 score after MI-LASSO regression. The mean of the MI-LASSO regression coefficients from 5 imputed datasets that predict GAD-7 severity are presented for both male and female study participants. Coefficients are sorted in descending order of magnitude.

Figure 2. Associations between socioeconomic status (SES) and GAD-7 score, stratified by race. Each panel describes the interaction of GAD-7 severity and SES risk score within each of 4 racial groupings. Column percentages were calculated as the counts within an individual cell divided by the total count of participants given that cells' GAD-7 severity score. Individual cells were shaded based on the column percentage; darker cells indicate a greater column percentage. Fisher Exact Tests for count data with simulated p-value were performed within each race; significant overall associations between SES risk score and GAD-7 severity for all races (White: $p<0.001$; Black or African American: $p=0.012$; Asian: $\mathrm{p}=0.010$; Other: $\mathrm{p}=0.02$ 
Females

Males Current smoker mory change Shortness of breath Absoughing up sputum

Age/ 10 years
Lightheadedness Lightheadedness
Other race Other race GERD Diarrhea Black race

Excessive belching or passing of gas Heat orpanic Nasal stuffiness Best available left ventricular EF

Numbness or loss of sensation

Days/30 with 10 hours of study watch wear Grip strength Aspartate aminotransferase (U/L)

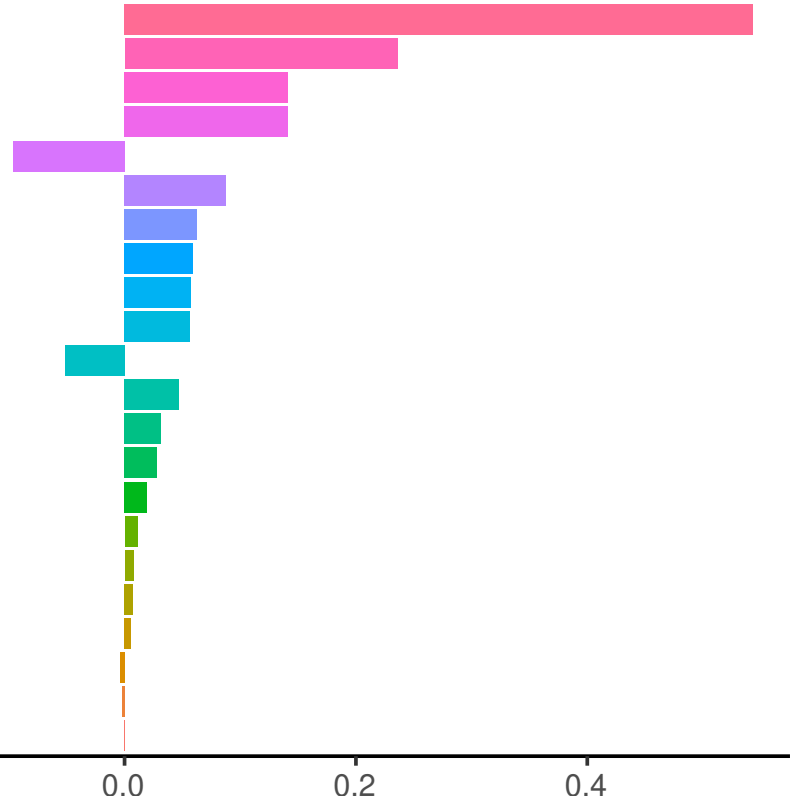

Mean of Penalized Regression

Coefficients from 5 Imputations

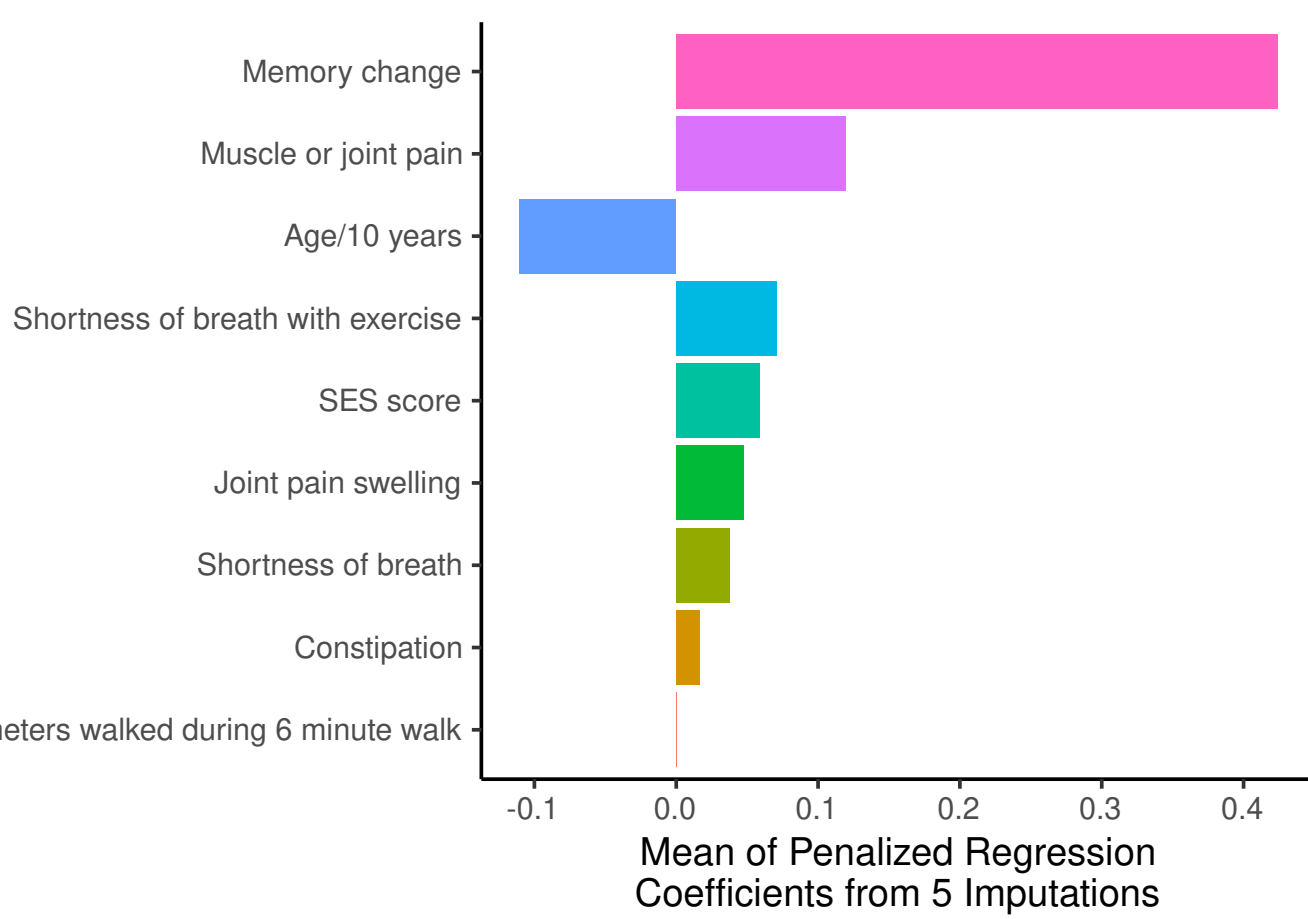



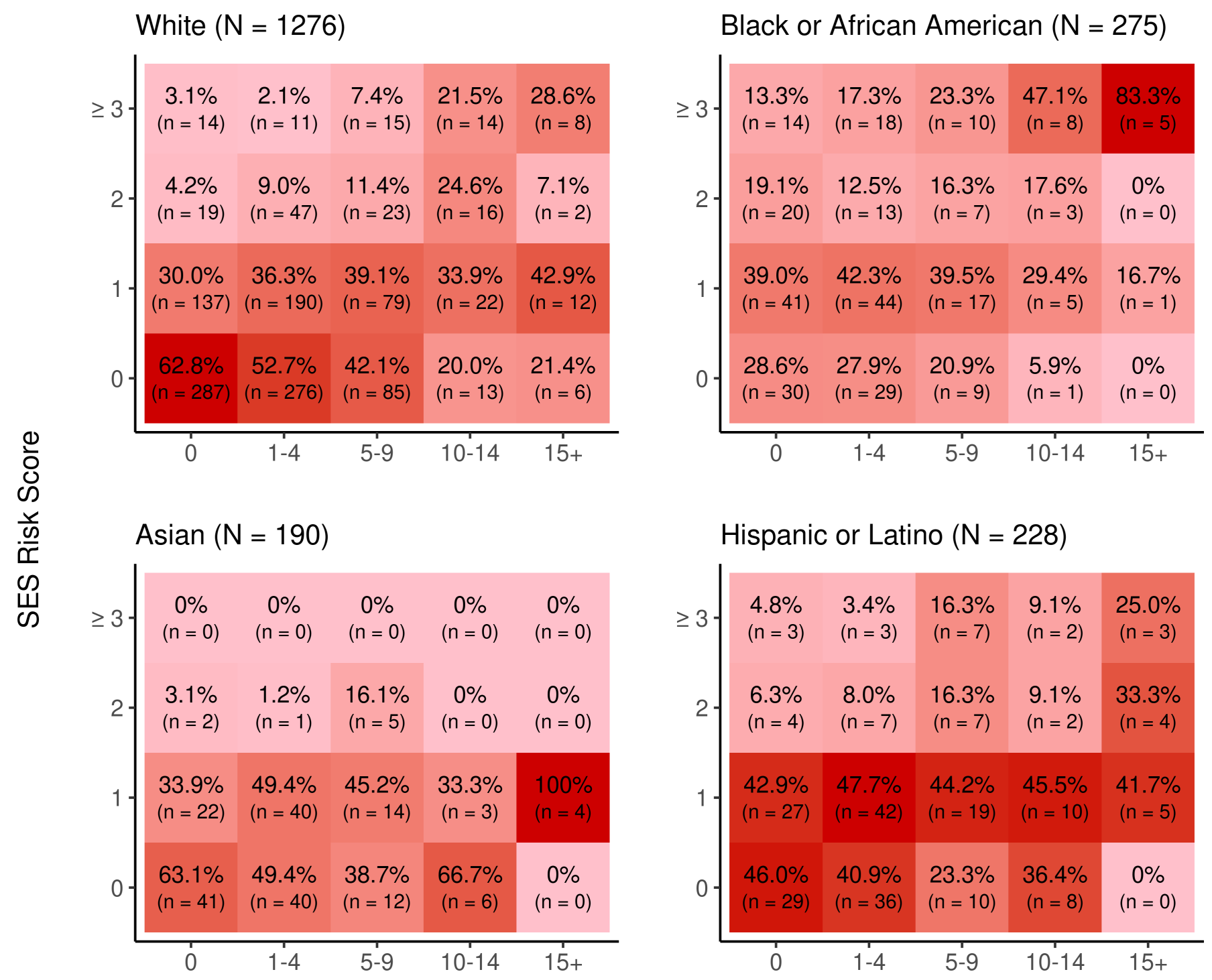

GAD-7 Severity 